Methods HVs and patients with reflux symptoms entered a prospective trial. Quality of life (RAND-36) and symptom severity (Eraflux) was assessed on and off PPI and after 2 weeks $\times 2$ /day PPI. Endoscopy recorded mucosal disease. Wireless pH system (Bravo ${ }^{\circledR}$, Given Imaging) measured acid reflux and symptoms up to 4 days. Receiver Operating Curve (ROC) assessed prediction of PPI response. For each prediction $80 \%$ of patients were randomly selected as training set, remaining $20 \%$ constituted test set. This was repeated 200 times producing average ROC with SEs. Area under Curve (AUC) quantified quality of prediction.

Results Complete data were available from 25/33 HVs (18F, age 20-56) and 70/108 patients (31F, age 18-77), >320 days in total. Oesophagitis was present in $9 \mathrm{HVs}$ (32\%: Grade A) and 26 patients (33\%: Grade $A=19, B=2, C-D=5$ ). Acid exposure time was elevated (AET $>5.6 \%$ ) in $3(12 \%) \mathrm{HVs}$ and $35(50 \%)$ patients. Eraflux off-PPI was $>25$ (consistent with GERD) in $60 / 63$ patients and fell by mean 7 (95\% CI 5 to 10) on PPI, 46\% reported positive response ( $>3$ fall). Diagnosis: Endoscopy, AET and reflux-symptom association analysis (Symptom Index (SI)) did not discriminate health/disease; but reflux-associated symptoms/day (nRS/Day) covered different ranges for $\mathrm{HV}$ and patients. Logistic regression with bootstrap validation identified that $\geq 3 \mathrm{RS} /$ day corresponded to $\sim 50 \%$ probability that participant was a patient.

PPI response: Clinical parameters and AET did not predict outcome. SI (9.2 vs 30.2, $\mathrm{p}=0.0023$ ) and nRS/Day (1 vs 2.6, $\mathrm{p}=0.012$ ) were higher in responders. RAND-36 scores for poor physical role and pain were higher in non-responders $(p \sim 0.1)$. SI ROC had an AUC of 0.73 (CI 0.51 to 0.92). SI $>25$ was the optimal cut-off for identifying PPI responders (Abstract PTU-197 figure 1). Prediction quality from $24 \mathrm{~h}$ studies was lower (AUC 0.69) and CIs for all parameters were wider with lower CI.

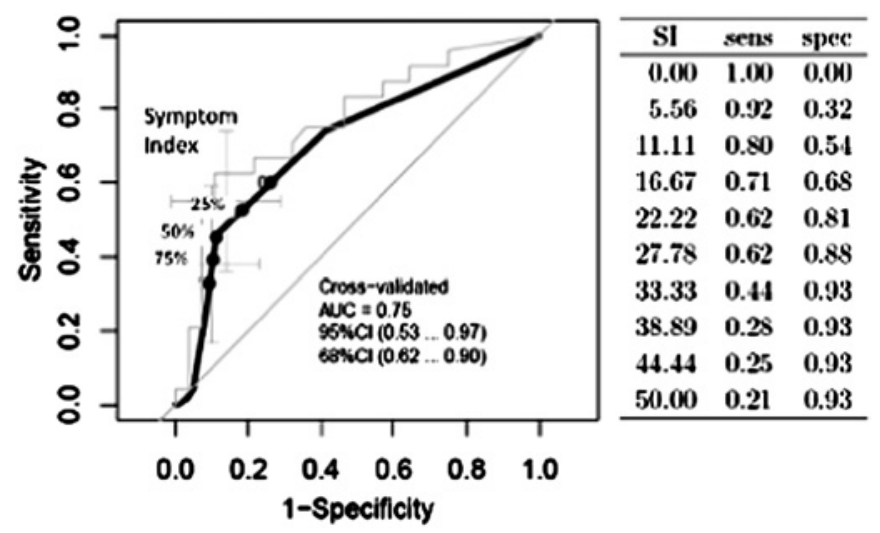

Abstract PTU-197 Figure 1 ROC for SI as predictor of PPI response. Error bars show SE. ROC with cross-validation is black line, without cross-validation is grey line ( $\sim 10 \%$ greater AUC).

Conclusion Diagnostic consistency for all parameters increases with study duration. A simple count of nRS/Day best discriminates HVs from patients on $\mathrm{pH}$ studies. SI $>25$ provides single best prediction of PPI response; but quality of predictions was modest in this population with low PPI response.

Competing interests None declared.

\section{PTU-198 TOWARDS OBJECTIVE ENDOSCOPIC DIAGNOSIS OF EARLY BARRETT'S NEOPLASIA USING FIBRE-OPTIC RAMAN SPECTROSCOPY}

doi:10.1136/gutjnl-2012-302514c.198

${ }^{1,2} \mathrm{M}$ Almond, ${ }^{*}{ }^{2} \mathrm{~J}$ Hutchings, ${ }^{2} \mathrm{C}$ Kendall, ${ }^{2} \mathrm{~N}$ Stone, ${ }^{3} \mathrm{~N}$ Shepherd, ${ }^{1} \mathrm{H}$ Barr. ${ }^{1}$ Oesophagogastric Surgery, Gloucestershire Royal Hospital, Gloucester, UK; ${ }^{2}$ Biophotonics
Research Unit, Gloucestershire Royal Hospital, Gloucester, UK; ${ }^{3}$ Department of Pathology, Gloucestershire Royal Hospital, Gloucester, UK

Introduction Raman spectroscopy is a powerful analytical technique that can rapidly and accurately identify biochemical changes in cells that have become neoplastic. We are aiming to translate this laboratory technique into an endoscopic tool that can identify highgrade dysplasia (HGD) and early malignant change (T1a, T1sm1) within Barrett's oesophagus. Here we aim to demonstrate that a novel fibre-optic Raman probe can correctly classify the pathology of ex vivo oesophageal tissue.

Methods A custom-built Raman probe, designed to fit through the instrument channel of a standard endoscope, was used to measure Raman spectra from ex vivo oesophageal tissue following oesophagectomy, endoscopic resection, or point biopsy from patients with Barrett's oesophagus +/- neoplasia. 1s spectra were measured using a monochromatic $830 \mathrm{~nm}$ laser for excitation. Multivariate analysis was used to correlate Raman spectra with histopathological diagnosis and calculate probe accuracy.

Results 348 spectra were measured from ex vivo tissue from 28 patients. Fibre-optic Raman measurements were able to discriminate between HGD/adenocarcinoma and non-dysplastic Barrett's oesophagus (BO) with a sensitivity of $91 \%$ and specificity of $96 \%$.

Conclusion Fibre-optic Raman Spectroscopy could enable endoscopic targeting of early neoplastic lesions in the oesophagus facilitating potentially curative endoscopic resection. Preparation is underway for an in vivo pilot study.

Competing interests None declared.

\section{PTU-199 VARIABLE REPORTING AND DIAGNOSIS OF EOSINOPHILIC OESOPHAGITIS ACROSS THE UK: DATA FROM THE BSG NATIONAL DISEASE REGISTER}

doi:10.1136/gutjnl-2012-302514c.199

${ }^{1} \mathrm{M}$ S J Wilson, ${ }^{1} \mathrm{~A}$ Simpson, ${ }^{2} \mathrm{~J} 0$ Hayat, ${ }^{3} \mathrm{~J} \mathrm{H}$ Ellison, ${ }^{1} \mathrm{~S}$ E Attwood, ${ }^{2} \mathrm{~J}-\mathrm{Y}$ Kang ${ }^{1}$ General Surgery, North Tyneside General Hospital, North Shields, UK; ${ }^{2}$ Gastroenterology, St George's Hospital, London, UK; ${ }^{3}$ British Society Gastroenterology, London, UK

Introduction Eosinophilic oesophagitis (EoE), a disorder characterised by intermittent dysphagia, was first described over 20 years ago. The true prevalence of this condition is not known. In 2010, the British Society of Gastroenterology (BSG) established a National EoE Register. The aim was to determine the frequency and pattern of diagnosis in the UK, and to identify centres for future research and areas where few patients are diagnosed and hence educational input may be beneficial. We report the data collected from March 2010 to January 2012

Methods A web-based register was established under the direction of the Oesophageal section of the BSG. Data entry was voluntary, anonymised, available by open access and did not require membership of the BSG. Clinicians from each hospital entered patient data. The date of birth and first part of patients' postcodes were recorded for demographic purposes and to prevent data duplication. Details of the specialty that made the diagnosis, the duration and pattern of symptoms, the diagnostic criteria and any treatment given were also recorded.

Results Data relating to 315 patients, although incomplete in some cases, were available for analysis. There were 229 patients from five centres and 86 patients from 30 other centres. No patients were entered from 70 hospitals. Their age ranged from 0 to 85 years. There were 236 males (75\%) and 77 females (3:1). Symptom duration ranged from 0 to $\geq 25$ years. $249(79.0 \%)$ patients had $>15$ eosinophils per high power field (eos/hpf), 11 (4\%) had $<15$ eos/hpf with $55(17 \%)$ patients having no eosinophil count recorded. The 
main presenting complaints were: dysphagia in 250 patients $(79 \%)$, food bolus obstruction in 127 (40\%) and heartburn in 59 (19\%). Endoscopy appeared normal in $47(15 \%)$ or consistent with reflux oesophagitis in $25(8 \%)$. In the remaining $243(77 \%)$ there were specific signs suggestive of EoE: strictures in 43, Schatzki ring in 10, white exudates in 16 and rings, trachealisation or furrows in the remainder.

Conclusion This is one of the world's largest registers of EoE. Dysphagia or food bolus obstruction are the most common symptoms. Endoscopic abnormalities suggestive of EoE are common but $23 \%$ do not have endoscopic signs to suggest EoE and therefore biopsy of all patients with dysphagia should be performed regardless of endoscopic appearance. There is variable reporting of EoE in the UK. Several centres had sufficient patients to facilitate research on diagnosis and treatment. However it is uncertain, for those hospitals that did not enter any patients, whether no patients with EoE were diagnosed or whether patients were diagnosed but not entered into the register.

Competing interests None declared.

\section{PTU-200 HOME JEJUNOSTOMY FEEDING POST-OESOPHAGECTOMY: A CHANGE IN PRACTICE}

doi:10.1136/gutjnl-2012-302514c.200

${ }^{1} \mathrm{M}$ Fanning, ${ }^{*}{ }^{1} \mathrm{~A}$ Mc Hugh, ${ }^{1} \mathrm{C}$ Browne, ${ }^{2} \mathrm{~N}$ Ravi, ${ }^{2} \mathrm{~J}$ V Reynolds, ${ }^{1} \mathrm{~L}$ A Healy. ${ }^{1} \mathrm{Clinical}$ Nutrition, St. James's Hospital, Dublin, Ireland; ${ }^{2}$ Department of Surgery, St. James's Hospital, Dublin, Ireland

Introduction Early enteral nutrition support via a needle catheter jejunostomy (NCJ) is a safe and effective method of providing nutrition support post-oesophagectomy. In our specialist unit all patients have a NCJ placed at the time of surgery for post-operative nutrition support. Maintaining nutritional status in the months following oesophageal resection is very challenging and up to $82 \%$ of patients lose weight by their first outpatient (OPD) visit. ${ }^{1}$ To address this, patients are now routinely discharged on supplementary feeding until their first OPD visit (after 4 weeks) and receive a dietetic telephone review 1 week post discharge. The aim of this retrospective review was to assess the impact of home jejunostomy feeding on nutritional status, length of hospital stay and timing of the first OPD visit.

Methods All patients who had an oesophageal resection in the 12 -month period before and after the change in practice were

Abstract PTU-200 Table 1 Comparison of outcomes from discharge to first OPD visit

\begin{tabular}{llll}
\hline & $\begin{array}{l}\text { Non home feeding } \\
\text { group }(\mathbf{n}=\mathbf{2 1})\end{array}$ & $\begin{array}{l}\text { Home feeding } \\
\text { group }(\mathbf{n}=\mathbf{2 8})\end{array}$ & p Value \\
\hline Weight change $(\mathrm{kg})$ & & $-1.9 \pm 0.60$ & \\
Mean & $-2.7 \pm 0.75$ & $-1.1(-8.3-3.5)$ & 0.416 \\
Median & $-2.8(-2.3-1.8)$ & & 0.058 \\
Weight change (\%) & & $-2.5 \pm 0.9$ & 0.622 \\
Mean & $-3.1 \pm 0.9$ & $-1.7(-13.8-4.3)$ & 0.665 \\
Median & $-3.4(-12.5-3.0)$ & & \\
Length of hospital stay (days) & $25 \pm 3.15$ & 0.416 \\
Mean & $20 \pm 2.05$ & $20(10-74)$ & 0.283 \\
Median & $16(10-56)$ & & 0.375 \\
Discharge to first OPD visit (days) & $25 \pm 1.69$ & 0.036 \\
Mean & $22 \pm 2.8$ & $24(10-47)$ & \\
Median & $16(10-56)$ & & \\
\hline
\end{tabular}

Mean \pm SEM, median (range) considered for this study. Patients who underwent emergency or palliative resections, were participating in a clinical trial or had incomplete data were excluded. Dietetic records and the cancer database were reviewed and analysed using SPSS $®$. The medians were compared using Mann-Whitney U test.

Results Weight loss was observed in $69 \%$ of all patients from discharge to first OPD visit. Patients who were discharged on home feeding experienced less weight loss (See Abstract PTU-200 table 1). Length of stay was not significantly increased in the home feeding group, but the length of time to first OPD visit increased significantly by 8 days (See Abstract PTU-200 table 1). The majority $(82 \%)$ of patients were discharged on $500 \mathrm{ml}$ volume of a $1.2-1.5 \mathrm{kcal} / \mathrm{ml}$ enteral feed, providing an average $40 \%$ of calculated energy and $46 \%$ of protein requirements. Of the home feeding group $52 \%$ continued to require jejunostomy feeding after the first OPD visit

Conclusion Home jejunostomy feeding can be successful in reducing weight loss following oesphagectomy and is frequently required for longer than 1-month post discharge. The increase in telephone contact may have contributed to a delay in the timing of first OPD visit. Organisation of home feeding in hospital did not delay discharge.

Competing interests None declared.

\section{REFERENCE}

1. Ryan, et al. Clinical Nutrition 2006;25:386-93

\section{Endoscopy II}

\section{PTU-201 THE ENDOSCOPIC COST OF BARIATRIC SURGERY}

doi:10.1136/gutjnl-2012-302514c.201

H Steed, ${ }^{*}$ H Golar, S Manjunath. Gastroenterology Department, Walsall Manor Hospitals NHS, Walsall, UK

Introduction Complication rates of Roux-en-Y gastric bypass (REYGB) vary from centre to centre, but anastomotic stricture is the commonest and is managed in the majority by endoscopic pneumatic dilatation. This study aimed to assess the endoscopic costs of REYGB surgery.

Methods All patients undergoing REYGB surgery over a 29-month period were included (553) and were followed up retrospectively and prospectively for a minimum of 180 days to monitor for endoscopic procedures performed in relation to the REYGB.

Results 113 patients had 147 endoscopic procedures, including 65 pneumatic dilatations, at a cost to the endoscopy department of $£ 58077$ over a 29 month study period, with an average cost of $£ 2003$ a month or $£ 105$ per REYGB operation performed. One patient required three dilatations, two required six dilatations and the rest were dilated once, using a range of $11 \mathrm{~mm}$ to $16 \mathrm{~mm}$ balloons at the endoscopists discretion. Five patients had completely occluded anastomoses. The anastomotic stricture rate for the group was $11.39 \%$. Three patients had acute ulceration at the anastomosis, giving a symptomatic ulcer rate of $0.7 \%$. The delay in time from surgery to dilatation ranged from 4-5-6 days, with a mean of 94 days. $60 \%$ of anastomotic strictures occurred within 3 months, $78 \%$ within 4 months, $92 \%$ within 5 months and $95 \%$ within 6 months.

Conclusion If bariatric surgery is performed locally then endoscopy departments must expect to factor in, not only the costs of dealing with actual complications, but also the cost of investigating for potential complications. The average cost to the endoscopy department per REYGB procedure performed is $£ 105$. 